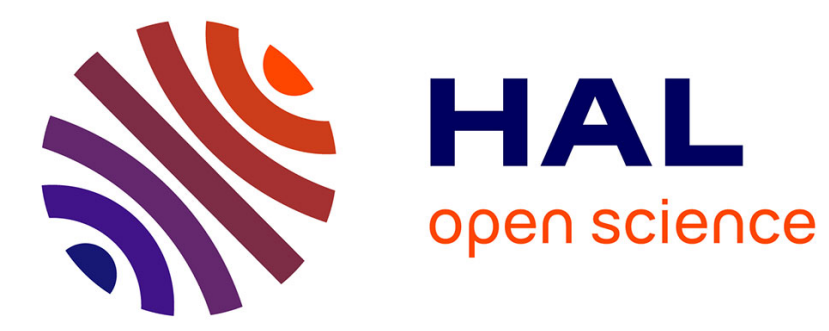

\title{
Modeling of current characteristics of segmented Langmuir probe on DEMETER
}

\author{
Nadia Imtiaz, Richard Marchand, Jean-Pierre Lebreton
}

\section{To cite this version:}

Nadia Imtiaz, Richard Marchand, Jean-Pierre Lebreton. Modeling of current characteristics of segmented Langmuir probe on DEMETER. Physics of Plasmas, 2013, 20 (5), pp.052903. 10.1063/1.4804336 . insu-01258109

\section{HAL Id: insu-01258109 \\ https://hal-insu.archives-ouvertes.fr/insu-01258109}

Submitted on 18 Jan 2016

HAL is a multi-disciplinary open access archive for the deposit and dissemination of scientific research documents, whether they are published or not. The documents may come from teaching and research institutions in France or abroad, or from public or private research centers.
L'archive ouverte pluridisciplinaire HAL, est destinée au dépôt et à la diffusion de documents scientifiques de niveau recherche, publiés ou non, émanant des établissements d'enseignement et de recherche français ou étrangers, des laboratoires publics ou privés.

\section{(ㅇ)(1) $\$$}

Distributed under a Creative Commons Attribution - NonCommercial - NoDerivatives $\mid 4.0$ 


\section{AIP | Physisos of}

\section{Modeling of current characteristics of segmented Langmuir probe on DEMETER}

Nadia Imtiaz, Richard Marchand, and Jean-Pierre Lebreton

Citation: Physics of Plasmas 20, 052903 (2013); doi: 10.1063/1.4804336

View online: http://dx.doi.org/10.1063/1.4804336

View Table of Contents: http://scitation.aip.org/content/aip/journal/pop/20/5?ver=pdfcov

Published by the AIP Publishing

\section{Articles you may be interested in}

Conditions for establishing quasistable double layers in the Earth's auroral upward current region

Phys. Plasmas 17, 122901 (2010); 10.1063/1.3520058

High-speed dual Langmuir probe

Rev. Sci. Instrum. 81, 073503 (2010); 10.1063/1.3455201

Understanding Langmuir probe current-voltage characteristics

Am. J. Phys. 75, 1078 (2007); 10.1119/1.2772282

Particle-in-cell Monte Carlo modeling of Langmuir probes in an Ar plasma

J. Appl. Phys. 97, 123310 (2005); 10.1063/1.1938275

How big is a small Langmuir probe?

Phys. Plasmas 7, 3084 (2000); 10.1063/1.874162

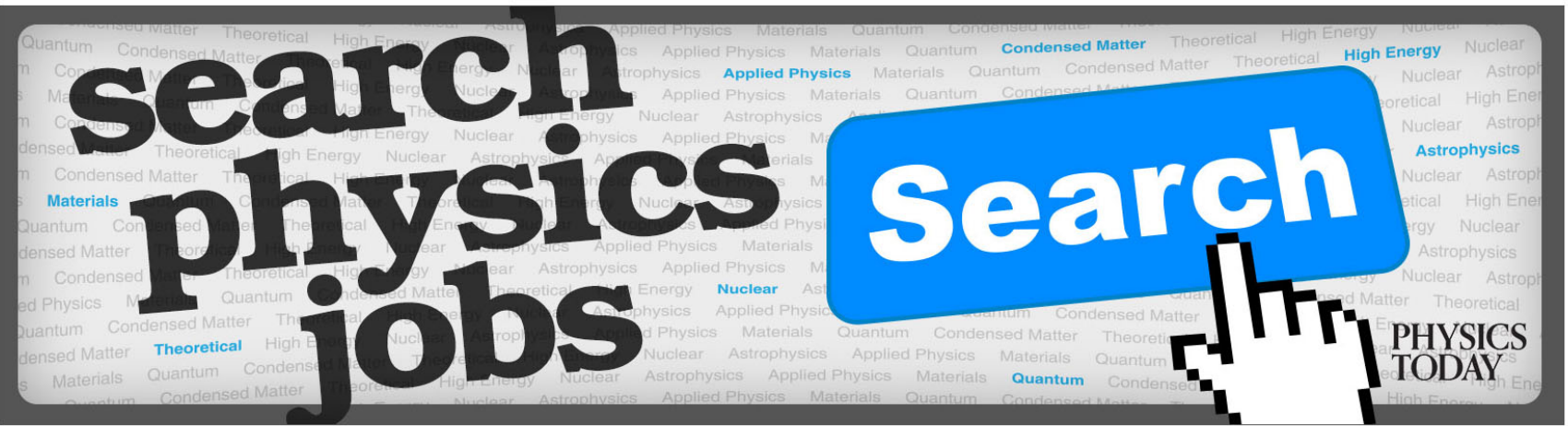




\title{
Modeling of current characteristics of segmented Langmuir probe on DEMETER
}

\author{
Nadia Imtiaz, ${ }^{1, a)}$ Richard Marchand, ${ }^{1, b)}$ and Jean-Pierre Lebreton ${ }^{2, c)}$ \\ ${ }^{1}$ Department of Physics, University of Alberta, Edmonton, Alberta T6G 2E1, Canada \\ ${ }^{2}$ Laboratoire de Physique et Chimie de l'Environnement et de l'Espace (LPC2E), \\ CNRS-Université d'Orléans, Orléans Cedex, France
}

(Received 4 March 2013; accepted 22 April 2013; published online 14 May 2013)

\begin{abstract}
We model the current characteristics of the DEMETER Segmented Langmuir probe (SLP). The probe is used to measure electron density and temperature in the ionosphere at an altitude of approximately $700 \mathrm{~km}$. It is also used to measure the plasma flow velocity in the satellite frame of reference. The probe is partitioned into seven collectors: six electrically insulated spherical segments and a guard electrode (the rest of the sphere and the small post). Comparisons are made between the predictions of the model and DEMETER measurements for actual ionospheric plasma conditions encountered along the satellite orbit. Segment characteristics are computed numerically with PTetra, a three-dimensional particle in cell simulation code. In PTetra, space is discretized with an unstructured tetrahedral mesh, thus, enabling a good representation of the probe geometry. The model also accounts for several physical effects of importance in the interaction of spacecraft with the space environment. These include satellite charging, photoelectron, and secondary electron emissions. The model is electrostatic, but it accounts for the presence of a uniform background magnetic field. PTetra simulation results show different characteristics for the different probe segments. The current collected by each segment depends on its orientation with respect to the ram direction, the plasma composition, the magnitude, and the orientation of the magnetic field. It is observed that the presence of light $\mathrm{H}^{+}$ions leads to a significant increase in the ion current branch of the I-V curves of the negatively polarized SLP. The effect of the magnetic field is demonstrated by varying its magnitude and direction with respect to the reference magnetic field. It is found that the magnetic field appreciably affects the electron current branch of the I-V curves of certain segments on the SLP, whereas the ion current branch remains almost unaffected. PTetra simulations are validated by comparing the computed characteristics and their angular anisotropy with the DEMETER measurements, as simulation results are found to be in good agreement with the measurements. @ 2013 AIP Publishing LLC. [http://dx.doi.org/10.1063/1.4804336]
\end{abstract}

\section{INTRODUCTION}

Langmuir probes (LPs) are widely used diagnostic instruments for both laboratory and space plasma. The technique consists of exposing an electrode to the plasma and measuring the collected current as a function of the bias voltage. By performing several measurements at different bias voltages, a current-voltage graph or characteristic curve is produced. The resulting characteristic curve of the probe is used to infer information about plasma parameters as, for example, the plasma density, temperature, plasma potential, and the floating potential. ${ }^{1-4}$

Early theoretical studies have been devoted to investigate the current characteristics of symmetrical probes, such as cylindrical, spherical, and planar probes. Mott-Smith and Langmuir proposed equations to derive the velocity distributions directly from the I-V curves of spherical and cylindrical probes in drifting Maxwellian plasma. ${ }^{5}$ Hoegy and Brace studied the response of planar, cylindrical, and spherical Langmuir probes

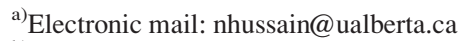

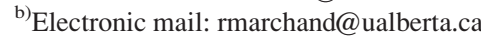

${ }^{\mathrm{c}}$ Electronic mail: jean-pierre.lebreton@cnrs-orleans.fr
}

in isotropic and anisotropic non-Maxwellian plasma. They found that, for isotropic distributions, the saturation current is temperature independent for cylindrical probes, whereas it is highly temperature dependent for the planar and spherical probes. Their study also showed that for isotropic plasma, these three geometries are equally suitable for the ionospheric electron density and the temperature measurements. For anisotropic distribution, however, these three probe geometries are not equally suitable for the measurement of ionospheric plasma parameters. ${ }^{6}$ El Saghir and Shannon presented integral relations for obtaining the electron energy distribution functions for different Langmuir probe geometries. ${ }^{7}$

For ionospheric applications, Langmuir probes have been widely used on satellites or rockets to measure electron and ion densities, temperatures and flow velocities, as well as spacecraft floating potentials in ionospheric and magnetospheric plasma. Many authors analyzed Langmuir probe measurements in space plasma to understand different physical phenomena. For example, Olson et al. applied a simple analytical probe-in-sheath model to the Langmuir probe data from Cassini spacecraft to study the spacecraft sheath effects on the current characteristics of the probe. ${ }^{8}$ Piel et al. studied the influence of the geomagnetic field and probe 
contamination on plasma diagnostics with Langmuir probes in the equatorial ionosphere. ${ }^{9}$ Some numerical models were also developed for the detailed description of Langmuir probes. For example, Hilgers et al. presented numerical results of SPIS (the spacecraft plasma interaction system) simulations of spherical and cylindrical Langmuir probe geometries in a regime in which the Orbit Motion Limited (OML) approximation is expected to be valid. ${ }^{10}$ The numerical results of their study are found to be in good agreement with theoretical predictions by Laframboise and the OML theory. ${ }^{11,12}$

Recently, the use of directional Langmuir probes to measure plasma flow velocity, and understand the dynamics of laboratory and space plasma has received special attention. Nagaoka et al., demonstrated experimentally and theoretically the measurements of the plasma flow velocity by using directional Langmuir probe under weakly ion-magnetized conditions. ${ }^{13}$ Lebreton et al., designed the DEMETER Langmuir probe experiment, also called Instrument Sonde de Langmuir (ISL), for in situ measurements of ionospheric plasma parameters. The ISL instrument consists of two sensors: a cylindrical $L P$ and a spherical segmented Langmuir probe (SLP). In their study, Lebreton et al. gave a brief description of the ISL instrument design, its in-flight operation, and data analysis techniques. ${ }^{14}$

Seran et al. developed a particle in cell (PIC) model to investigate the capabilities of the SLP to diagnose bulk plasma parameters. ${ }^{15}$ Their model, however, did not account for the influence of the magnetic field on current collection of the SLP. The goal of the present study is to simulate the current characteristics of the DEMETER SLP. For this purpose, we use PTetra which is capable of simulating the time dependent interaction of satellites with space plasma. ${ }^{16}$ The numerical results of our study show some interesting features. These include the angular anisotropy in the current collected by various collectors, the sensitivity of the electron current to the orientation of the magnetic field, and the enhancement in the ion current due to the presence of the minority light $\mathrm{H}^{+}$ions.

The remainder of this article is organized in the following manner: Sec. II briefly explains the design and the technique of the SLP. Section III describes the numerical approach used to compute the I-V curves of the SLP. In Sec. IV, we present example results and their comparison with DEMETER measurements. Finally, Sec. V contains a summary and some conclusions.

\section{THE DEMETER SLP}

The SLP is part of the scientific payload of a French microsatellite, DEMETER (Detection of Electro-Magnetic Emissions Transmitted from Earthquake Regions). A complete description of the segmented Langmuir probe is given by Lebreton $e t$ al. ${ }^{14}$ The SLP is part of the ISL instrument that comprises two Langmuir probes: a cylindrical Langmuir probe and the SLP as shown in Figure 1. DEMETER was used as a flight demonstrator for the SLP. The cylindrical Langmuir probe was regularly used to measure bulk parameters of the ionospheric plasma, while the SLP was only used occasionally, as the primary objective was to characterize its performance in different plasma conditions encountered during the DEMETER mission. The SLP consists of a spherical probe with its surface divided into seven independent collectors; six spherical segments each of radius $0.5 \mathrm{~cm}$ and a guard electrode. The guard electrode consists of a sphere of radius $2 \mathrm{~cm}$ and a small cylinder $(1.5 \mathrm{~cm}$ in length, $6 \mathrm{~mm}$ in radius). The six segments are electrically insulated from each other and from the guard electrode, but all components of the SLP are kept at the same potential. They are positioned around the sphere at different angles with respect to one another. The orientation of the segments around the sphere is illustrated in Figure 1. Segment $S_{2}$ is aligned with the satellite velocity (along Z-axis in the ram direction). Other segments $S_{1}, S_{3}$ are located symmetrically at an angle of $45^{\circ} ; S_{4}, S_{5}$, and $S_{6}$ are at an angle $90^{\circ}$ with respect to the ram direction. The probe is mounted on a post of $6 \mathrm{~cm}$ in length and $4 \mathrm{~mm}$ in radius. On DEMETER, this post is grounded with respect to the spacecraft body. In the simulations, it is used as a reference with respect to which the probe bias is determined. The I-V characteristics are obtained by applying the same potential to all seven collectors and measuring the current collected by each segment individually.

\section{NUMERICAL APPROACH}

A three-dimensional PIC code PTetra is used to simulate the current-voltage characteristics of the probe. The model is based on a fully kinetic description of all plasma species with physical charges and masses. The model accounts for a uniform and constant background magnetic field. It also accounts for several phenomena of physical importance in the spacecraft interaction with the plasma. These include satellite charging, photoelectrons, and secondary electrons emission. For Low
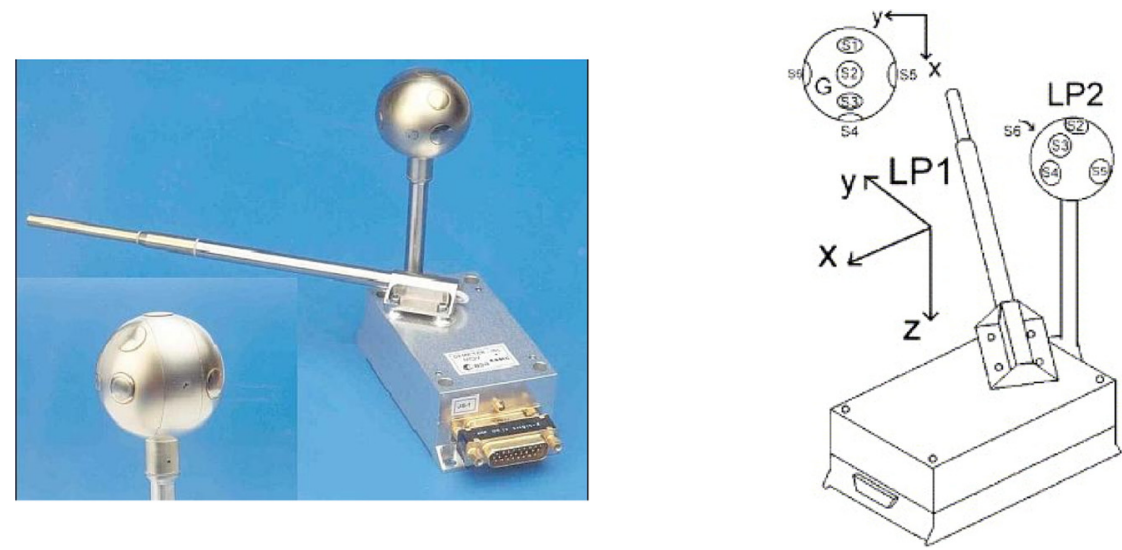

FIG. 1. Segmented Langmuir probe (left) and illustration of the various segments (right). Reprinted with permission from Lebreton et al., Planet. Space Sci. 54, 472 (2006). Copyright 2006 Elsevier. 
Earth Orbit (LEO) satellites (at the altitude range $100 \mathrm{~km}$ to $1000 \mathrm{~km}$ in the ionosphere), the impact of the secondary electrons is not significant. However, the photoemission can affect the current collection of the sunlit area which eventually depends on the solar angle. In this study, the effect of photoelectrons was assessed by comparing the characteristics computed with and without photoemission. The differences were found to be small $(<5 \%)$. A more rigorous analysis of photoemission effects would require the inclusion of a more complete description of the satellite geometry, such as the satellite body and the solar panel. Such a study is beyond the scope of the present paper.

PTetra uses a standard PIC approach, in which simulation particles, or macroparticles, represent many physical particles. In addition to charge and mass, each macroparticle also carries a statistical weight corresponding to the number of physical (real) particles that it represents. In this study, $10^{8}$ macroparticles are used in the simulation domain, to simulate the plasma near the probe. The mesh used in the study is unstructured with tetrahedral elements. It was generated with the mesh generator CUBIT $^{17}$ and it consists of 79861 vertices connected in 454950 tetrahedra. The resolution of the mesh varies in space in order to resolve the fine features near the probe. The size of largest elements in the simulation domain is $2 \mathrm{~cm}$ and the smallest ones on the probe is $2.5 \mathrm{~mm}$. The outer boundary of the simulation domain is located sufficiently far from the probe for the boundary conditions to have a negligible influence on the simulation results. The equations of motion of the particles are solved by using second order accurate leap frog method. The integration of these equations requires the knowledge of $\vec{E}$ and $\vec{B}$ fields. The magnetic field $\vec{B}$ is calculated from the International Geomagnetic Reference Field (IGRF) model $^{18}$ by using the time and satellite position at which measurements were made. Poisson's equation is solved as a boundary value problem with Dirichlet boundary conditions. ${ }^{16}$ The numerical solution of Poisson's equations requires the volume charge density and the charge collected by each probe component. A collected current is specified on the probe, whereas the post is assumed to be floating. Note that, other than the post and the spherical probe, our simulations do not account for the other components of the satellite. If the entire spacecraft were included in the model, the negative of the current imposed on the probe would also have to be imposed on the rest of the satellite in order to ensure current balance. In such a case the post would only be collecting a small fraction of that current, owing to its small surface area compared to that of a spacecraft body. This is why the post, used as a satellite ground proxy, is assumed to be collecting zero net current. The imposed probe collected current is varied in such a way as to produce a potential difference with the (grounded) post ranging form $-5 \mathrm{~V}$ to $+5 \mathrm{~V}$. The simulation parameters used in our analysis and summarized in Table I correspond to the DEMETER measurements along day side orbit 06911-0.

\section{RESULTS AND DISCUSSION}

In this section, the computed characteristics of the SLP and their comparison with the DEMETER satellite
TABLE I. Physical parameters assumed in the reference simulation, corresponding to DEMETER orbit 06911-0 at 8:16 UT.

\begin{tabular}{lc}
\hline \hline Physical parameter & Value \\
\hline Electron density & $1.3 \times 10^{10} \mathrm{~m}^{-3}$ \\
Plasma temperature & $0.3 \mathrm{eV}$ \\
Magnetic field $\left(\vec{B}_{o}\right)$ & $(40.68,-1.257,-6.787) \mu \mathrm{T}$ \\
Plasma flow velocity $\left(v_{d}\right)$ & $(0,0,7500) \mathrm{m} / \mathrm{s}$ \\
Altitude & $712 \mathrm{~km}$ \\
Geographic latitude & $70.76^{\circ} \mathrm{N}$ \\
Geographic longitude & $54.40^{\circ}$ \\
Majority ions & $78 \% \mathrm{O}^{+}$ \\
Minority ions & $22 \% \mathrm{H}^{+}$ \\
Debye length & $0.035 \mathrm{~m}$ \\
Electron thermal velocity $v_{t h e}=\sqrt{\frac{2 k_{B} T_{e}}{m_{e}}}$ & $324.8 \mathrm{~km} / \mathrm{s}$ \\
Electron thermal gyro radius & $0.042 \mathrm{~m}$ \\
Hydrogen ion thermal velocity $v_{t h H}=\sqrt{\frac{2 k_{B} T_{i}}{m_{H}}}$ & $7.6 \mathrm{~km} / \mathrm{s}$ \\
Oxygen ion thermal velocity $v_{t h o}=\sqrt{\frac{2 k_{B} T_{i}}{m_{O}}}$ & $1.9 \mathrm{~km} / \mathrm{s}$ \\
Ion thermal gyro radius & $5.4 \mathrm{~m}$ \\
\hline
\end{tabular}

measurements are presented. Three simulation cases are considered to study the influence of different physical phenomena on the current collection of the SLP. These are as follows:

- A reference case, corresponding to the plasma composed of $22 \% \mathrm{H}^{+}$and $78 \% \mathrm{O}^{+}$ions with a magnetic field obtained from the IGRF model. ${ }^{18}$
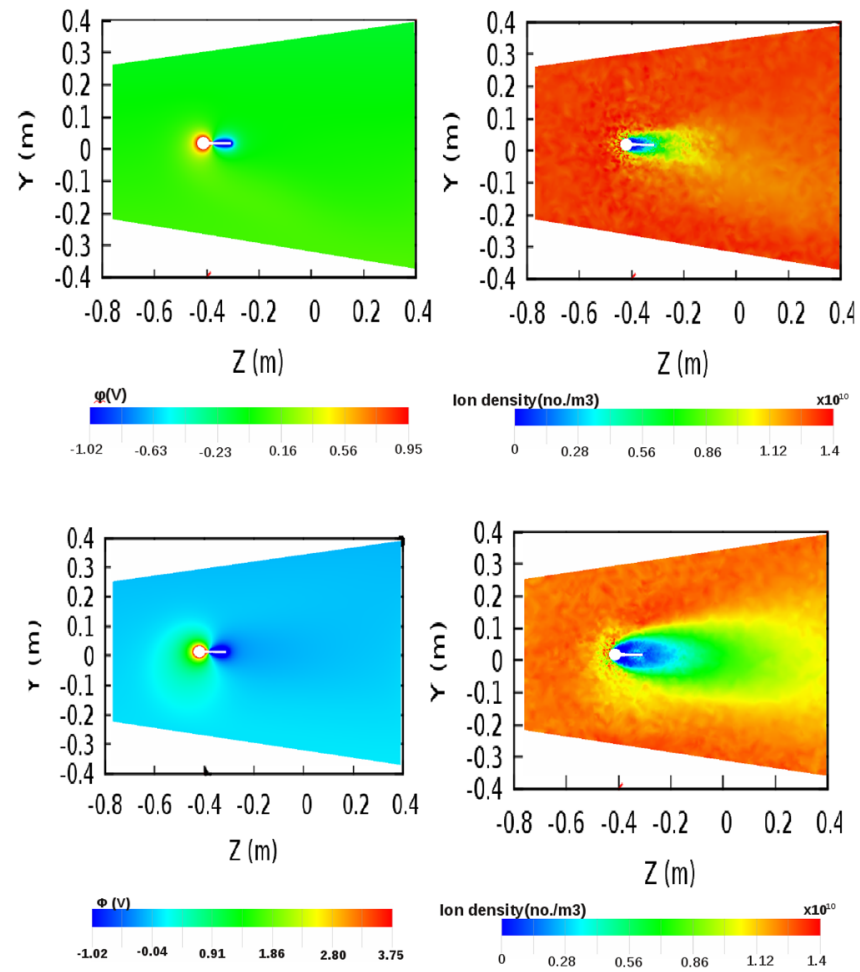

FIG. 2. Profiles of the sheath potential (left) and the corresponding wake in ion density behind the probe (right). Plasma is drifting in the $\mathrm{Z}$ direction, whereas the orbital velocity of the satellite is in the $-\mathrm{Z}$ direction. 
- A plasma with $100 \% \mathrm{O}^{+}$ions. This case is presented to show the effect of light $\left(\mathrm{H}^{+}\right)$and heavy $\left(\mathrm{O}^{+}\right)$ions on the ion current branch of the SLP.

- A case in which the magnetic field is varied in magnitude and direction with respect to the reference magnetic field $\left(\vec{B}_{o}\right)$. This is done to illustrate the sensitivity of the collected electron current to the orientation and the magnitude of the magnetic field.

The simulation results obtained for these three cases are compared with in-flight DEMETER satellite measurements. Two sweeps are presented for the measurements: a short duration (fast) and a long duration (slow) sweep. These two sweeps are explained by Lebreton et al. ${ }^{14}$ in Sec. III B 5 . The data acquisition times for the fast and the slow sweeps are $0.25 \mathrm{~s}$ and $2 \mathrm{~s}$, respectively. Each sweep consists of two parts: an up (negative to positive) and a down scan (positive to negative). In Figures 3 and 4, the fast up scans (thick solid line) are followed by the fast down scan (broken line), and the slow up scans (thin solid) are followed by slow down scans (broken thin line). It is noted that the up and down scans for both sweeps show hysteresis. The difference between the I-V curves for the up and the down sweeps is used to estimate the surface properties of the probe, and in particular to characterize the thin layer due, for example, to surface contamination, or perhaps to a thin dielectric film of unknown origin, which is thought to be responsible for the hysteresis which varies with sweep speed. The characteristics of the properties of the layer and its influence on the determination of the plasma parameters are the subject of ongoing work (J.-P. Lebreton, private communication, 2012). ${ }^{20}$
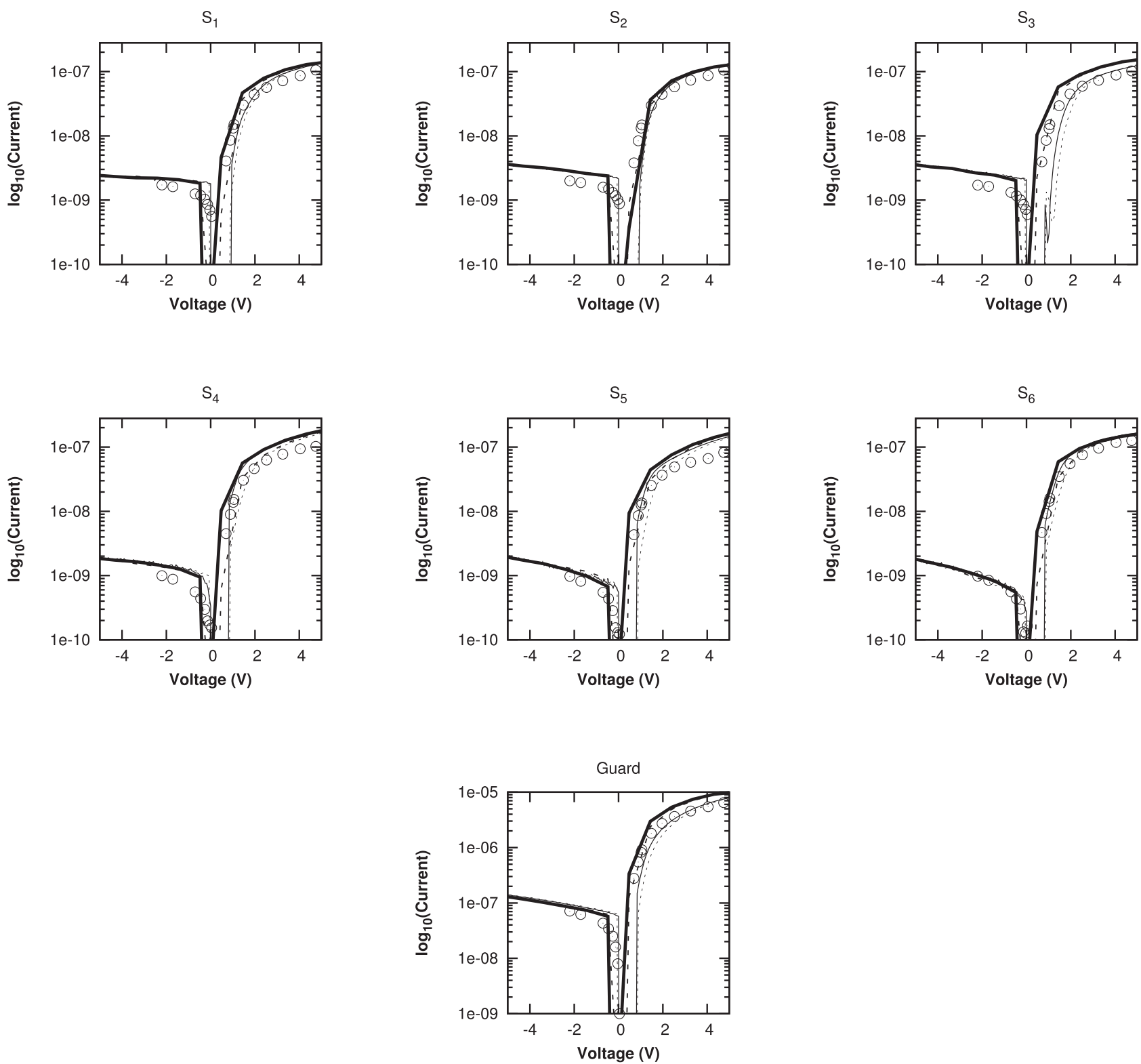

FIG. 3. Comparison between the simulated and measured I-V curves for segments $S_{1}, S_{2}, S_{3}, S_{4}, S_{5}, S_{6}$ and the guard electrode. The thicker lines show the characteristics for the fast scan and thinner lines show the characteristics for the slow scan. In both cases, the solid lines are for the up scan (negative to positive) and dashed lines for the down scan (positive to negative). Circles show the characteristics obtained from the reference case. 


\section{A. Reference case}

The reference conditions correspond to the plasma parameters that are given in Table I. The composition of the ionospheric plasma for the LEO environment depends strongly on the altitude, latitude, local time, and the solar activity. The ion composition on board DEMETER is obtained from the ion composition measurements of the Instrument d'Analyse du Plasma (IAP). ${ }^{19}$ The simulations are done with the same density of $\mathrm{H}^{+}$and $\mathrm{O}^{+}$ions as measured on DEMETER; that is, $22 \% \mathrm{H}^{+}$and $78 \% \mathrm{O}^{+}$. The orbital velocity of the satellite is in the Z-direction and in the probe frame of reference; the plasma is drifting with the same speed in the opposite direction. The plasma drift velocity is 5 times greater than the $\mathrm{O}^{+}$thermal velocity $(1.9 \mathrm{~km} / \mathrm{s})$, and is comparable to the $\mathrm{H}^{+}$thermal velocity $(7.6 \mathrm{~km} / \mathrm{s})$. The Mach number defined as the plasma drift velocity divided by the ion sound speed $\left(M=\frac{v_{d}}{c_{a}}\right)$ is approximately 5 . The plasma flow is supersonic and a visible wake in the ion density is expected behind the probe in all cases. The structure of the wake region is shown in Figure 2 for the weakly biased $(+2 \mathrm{~V})$ and strongly biased $(+5 \mathrm{~V})$ probe. It is observed that the size of the wake region and plasma sheath potential vary with the probe biasing. The current collected by the seven segments of the SLP as a function of bias voltage is computed with PTetra. The resulting characteristics in the range $-5 \mathrm{~V}$ to $+5 \mathrm{~V}$ are illustrated in Figures 3 and 4 . The anisotropy in the ion current collected by the various segments shows that the segment $S_{2}$ (facing the ram direction) collects slightly more current than the other segments. The anisotropy in the collected ion current is mainly controlled by the direction of the plasma flow velocity; hence, the segment facing the ram directly collects the largest current. The comparison of the reference simulation results (accounting for
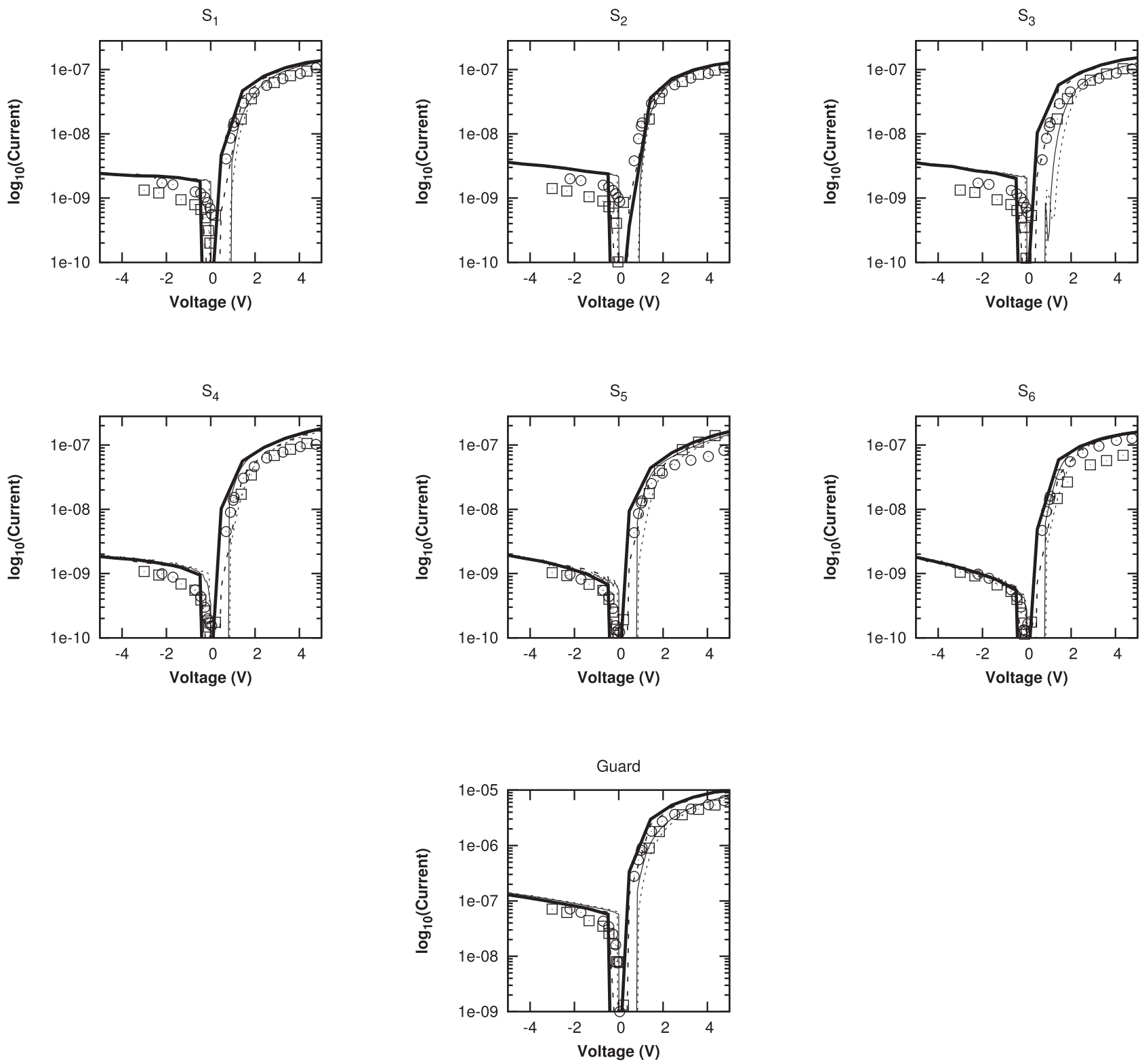

FIG. 4. Comparison between the measured and simulated I-V curve of segments $S_{1}, S_{2}, S_{3}, S_{4}, S_{5}, S_{6}$ and the guard electrode, showing the effect of the ion composition. The measured characteristics and the reference case are the same as in Figure 3. Circles represent the characteristics obtained with the reference case and squares show the characteristics computed with $100 \% \mathrm{O}^{+}$ions. 
the $\mathrm{H}^{+}$ions) with DEMETER measurements shows good qualitative and quantitative agreement in both ion and electron current branch of the I-V curves. A proper calibration has been made for the I-V converter of the cylindrical probe and for seven I-V converters of the SLP prior to launch. The description of the calibration is beyond the scope of the present study. However, the post-flight calibration of the I-V converters has not been available yet. This could be the source of uncertainty in the ion current part of the I-V curve. It could also affect the electron part of the characteristic, but it would likely be negligible. There are various physical factors that play an important role in the current collection of the SLP as for example, the ionic composition of the ionospheric plasma, the attitude of the satellite and the orientation of the magnetic field. Numerically, it is straightforward to include each effect separately in order to assess its relative importance. The resulting variation in the current collected by the SLP is studied for the presence of heavy ions and with
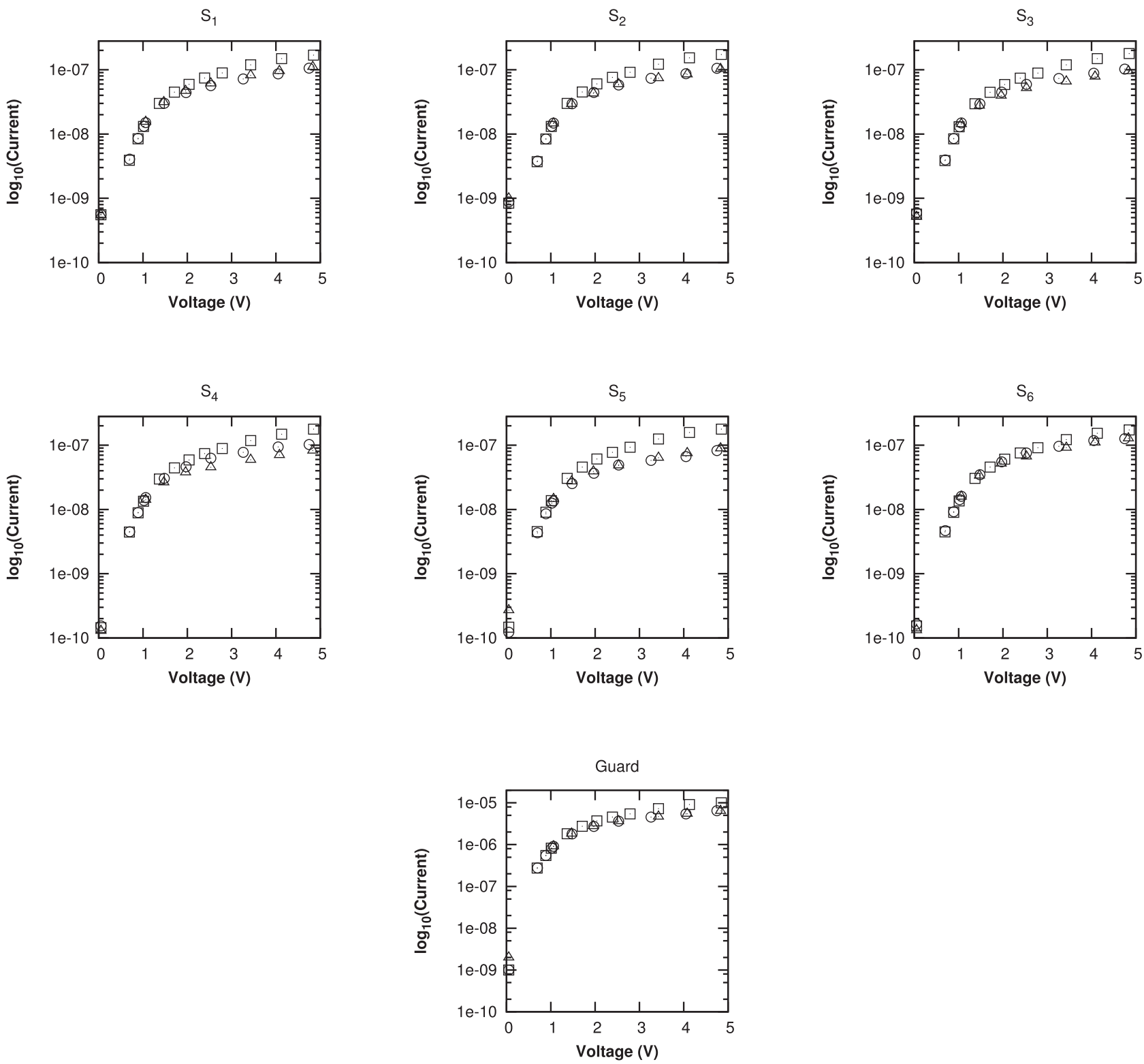

a small variation in the orientation of the magnetic field associated with a small error in the satellite attitude. These two effects are considered below.

\section{B. Ion mass and composition}

The effect of ion mass and composition is assessed by comparing the simulation results obtained for a $100 \% \mathrm{O}^{+}$ plasma, with the measurements and with the simulation results obtained for the reference case. This comparison is shown in Figure 4. It is observed that the simulated I-V curves show qualitative agreement with the measurements. Quantitatively, however, there are marked discrepancies in the ion current branch. It is found that in an assumed $100 \%$ $\mathrm{O}^{+}$plasma, the ion current collected in negative biasing significantly less (by $\sim 36 \%$ ) than that in the reference case. This decrease in the ion current can be understood from the OML theory of the spherical probes. In this case, the Debye

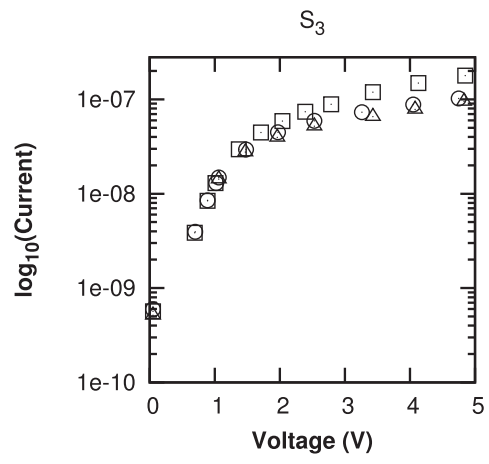


length $\left(\lambda_{D}=0.035 \mathrm{~m}\right)$ is greater than the probe radius $(a=0.02 \mathrm{~m})$ and the sheath around the SLP is thick. The motion of the ions in the thick sheath depends on the impact parameter. The ions approaching the probe with distances exceeding the impact parameter $\left(b_{c}\right)$ do not contribute to the probe current. Only ions which have approaching distances smaller than the impact parameter will contribute to the collected current. The impact parameter depends on the ratio of the probe potential to the bulk energy of the ions. The bulk energies of the $\mathrm{H}^{+}$and $\mathrm{O}^{+}$ions are $0.29 \mathrm{eV}$ and $4.7 \mathrm{eV}$, respectively. The corresponding impact parameter $b_{c}$ of the $\mathrm{H}^{+}$ions is 3 times larger than that of the $\mathrm{O}^{+}$ions. Therefore, the effective cross section $\left(\sigma=\pi b_{c}^{2}\right)$ of $\mathrm{H}^{+}$is 9 times that of the $\mathrm{O}^{+}$ions. The resulting current collected by the probe immersed in a plasma made of $100 \% \mathrm{O}^{+}$ions therefore, will be less than that of plasma containing lighter $\mathrm{H}^{+}$ions.

\section{Sensitivity to the magnetic field}

The magnetic field also affects the I-V curves of the SLP. In the reference case, the magnetic field $\vec{B}_{o}=(40.68,-1.257$, $-6.787) \mu \mathrm{T}$ is obtained from the IGRF model. The angle between the $\vec{B}_{o}$ and the Y-axis (normal to segments $S_{5}$ and $S_{6}$ ) is $88.23^{\circ}$ which corresponds to an angle of $\sim 2^{\circ}$ with respect to the surface of the top segment. The reference $\vec{B}_{o}$ is almost parallel (grazing) to segments $S_{5}$ and $S_{6}$. In order to determine the magnetic sensitivity of the results to the orientation of the magnetic field, the simulations are repeated with a magnetic field $\vec{B}^{\prime}=(29.17,29.17,0.78) \mu \mathrm{T}$. The magnitude of $\vec{B}^{\prime}$ is the same as that of the reference magnetic field, but with a change in direction by $\sim 45^{\circ}$. Current characteristics are also computed with zero magnetic field. The resulting characteristics for the above mentioned two cases are compared with the reference simulation case and are illustrated in Figure 5. In this case, magnetization plays an important role in the interpretation of the I-V curves of the SLP. It is a parameter used to specify the ability of the magnetic field to affect the trajectories of the particle. It is the ratio between the thermal gyro radius of the particle and the characteristic scale length of the system. If this ratio is small $(\leq 1)$ particles are magnetized; otherwise, they are unmagnetized. In our study, the scale length is characterized by the radius of the probe $0.02 \mathrm{~m}$, whereas the thermal gyro radii of electrons and $\mathrm{O}^{+}$ions are $0.042 \mathrm{~m}$ and $5.4 \mathrm{~m}$, respectively. Whence, electrons here are weakly magnetized and ions are effectively unmagnetized. Varying the orientation of the magnetic field by $45^{\circ}$ has relatively little effect on most characteristics. In the absence of the magnetic field, however, the differences in the collected electron current are noticeable. It is observed that with zero magnetic field, the SLP simulated collected electron current is approximately twice the value computed with the reference case.

\section{CONCLUSIONS}

The PTetra numerical model is well adapted to compute the current characteristics of a segmented Langmuir probe, which is part of the scientific payload of the DEMETER microsatellite. Three cases were considered to study the sensitivity of the I-V curves: The first one serves as a reference with respect to which the other two cases are compared. The second case looks at the effect of the ion mass composition, and the third case shows the sensitivity of the electron collected current to the magnetic field. PTetra simulation results are compared with DEMETER measurements along a day side orbit. The comparison shows good qualitative agreement in all cases. In the reference case, that accounts for $78 \% \mathrm{O}^{+}$and $22 \% \mathrm{H}^{+}$, the collected current is comparable to the measured current with some minor differences. There are several physical factors at play in the current collection of the SLP. These include the ionospheric plasma composition, the strength, and orientation of the magnetic field. A quantitative assessment of these effects was made by carrying out simulations in which each effect was taken into account separately. The high mobility of the $\mathrm{H}^{+}$ions leads to a significant contribution in the ion collected current when the probe is negatively biased. The probe collects 3 times more ion current compared to the computed collected currents in an assumed $100 \% \mathrm{O}^{+}$plasma. The moderate magnetization of the electrons causes the electron current branch to be sensitive to the presence of the magnetic field. In the absence of the magnetic field, there is a significant increase in the electron current collected by all segments compared to the reference case. However, the magnetic field does not affect the ion current branch. This is due to the fact that collected electrons, even when weakly magnetized, are constrained to come from the magnetic flux tube of radius equivalent to the electron thermal gyro-radius $(\sim 4 \mathrm{~cm})$. When $\mathrm{B}=0$, on the other hand, the collected electrons come from all directions without any constraint on their motion and contribute significantly to the electron current branch. The best agreement with the measurements is obtained with the reference case, which accounts for the magnetic field and the plasma made of $78 \% \mathrm{O}^{+}$and $22 \% \mathrm{H}^{+}$ions as measured on DEMETER. These effects, therefore, need to be taken into account in the interpretation of the I-V curves of a segmented Langmuir probe.

\section{ACKNOWLEDGMENTS}

N.I. and R.M. acknowledge the support by Natural Sciences and Engineering Research Council of Canada (NSERC). The DEMETER Langmuir probe instrument was developed, and funding provided as part of the Instrument research programme, while J.-P.L. was affiliated with the Space Science Department of ESA at ESTEC. This work was also supported in part by the International Space Science Institute in Bern, Switzerland. The simulations presented in this work made use of the WestGrid computing facilities.

${ }^{1}$ J. M. Díaz-Cabrera, M. V. Lucena-Polonio, J. I. Palop, R. M. Crespo, M. A. Hernández, A. Tejero-del-Caz, and J. Ballesteros, Appl. Phys. 111, 063303 (2012).

${ }^{2}$ F. F. Chen, Plasma Sources Sci. Technol. 21, 055013 (2012).

${ }^{3}$ R. Merlino, Am. J. Phys. 75, 1078 (2007).

${ }^{4}$ J. M. Zhang, L. Hong, C. Zhi-Peng, L. Chen, X. Jin-Lin, L. Tao L. A-Di, and L. Wan-Dong, Chin. Phys. Lett. 29, 075201 (2012).

${ }^{5}$ H. M. Mott-Smith and I. Langmuir, Phys. Rev. 28, 727 (1926). 
${ }^{6}$ R. W. Hoegy and H. L. Brace, Rev. Sci. Instrum. 70, 3015 (1999).

${ }^{7}$ A. El Saghir and S. Shannon, Plasma Sources Sci. Technol. 21, 025003 (2012).

${ }^{8}$ J. Olson, N. Brenning, J.-E. Wahlund, and H. Gunell, Rev. Sci. Instrum. 81, 105106 (2010).

${ }^{9}$ A. Piel, M. Hirt, and C. T. Steigies, J. Phys. D 34, 2643 (2001).

${ }^{10}$ A. Hilgers, S. Clucas, B. Thiebault, J. F. Roussel, J. C. Mateo-Velez, J. Forest, and D. Rodgers, IEEE Trans. Plasma Sci. 36, 2319 (2008).

${ }^{11}$ J. G. Laframboise, UTIAS Report No. 100, Institute for Aerospace Studies, University of Toronto, 1966.

${ }^{12}$ J. G. Laframboise and L. J. Sonmor, J. Geophys. Res. doi: 10.1029/ 92JA00839 98, 337 (1993).

${ }^{13}$ K. Nagaoka, K. Hara, T. Ishihara, A. Okamoto, S. Yoshimura, and M. Y. Tanaka, J. Phys. Soc. Jpn. 70, 131 (2001).
${ }^{14}$ J.-P. Lebreton, S. Stverak, P. Travnicek, M. Maksimovic, D. Klinge, S. Merikallio, D. Lagoutte, B. Poirier, P. L. Blelly, Z. Kozacek, and M. Salaquarda, Planet. Space Sci. 54, 472 (2006).

${ }^{15}$ E. Seran, J. J. Berthelier, F. Z. Saouri, and J. P. Lebreton, Ann. Geophys. 23, 1723 (2005).

${ }^{16}$ R. Marchand, IEEE Trans. Plasma Sci. 40, 217 (2012).

${ }^{17}$ See www.cubit.sandia.gov/cubitprogram.html for the description of two and three dimensional finite element mesh generator.

${ }^{18}$ See http://www.geomag.bgs.ac.uk/dataservice/modelscompass/igrf.html for more information about IGRF Model (11th Generation).

${ }^{19}$ J. J. Berthelier, M. Godefroy, F. Leblanc, E. Seran, D. Peschard, P. Gilbert, and J. Artru, Planet. Space Sci. 54, 487 (2006).

${ }^{20} \mathrm{~J}$. P. Lebreton, "On the issue of surface contamination of a Langmuir Probe sensor: Demeter ISL results," J. Geophys. Res. (to be published). 